\title{
Prospects for planet formation in multiple stellar systems
}

\author{
Gaspard Duchêne $e^{1,2}$ \\ ${ }^{1}$ Astronomy Department, UC Berkeley, 601 Campbell Hall, Berkeley CA 94720-3411, USA \\ email: gduchene@berkeley.edu \\ ${ }^{2}$ Laboratoire d'Astrophysique de Grenoble, CNRS/UJF UMR 5571, 414 rue de la Piscine, \\ BP 53, 38041 Grenoble Cedex 9, France
}

\begin{abstract}
As I review here, planet formation in multiple stellar systems is far from exceptional. However, it appears that binaries with projected separation in the 5-100 AU range have different initial conditions and end result properties than wider systems, probably because they undergo different physical processes. In addition, very tight binaries, with projected separation less than a few AU, seem to fulfill all the known requirements to form planetary systems, suggesting that circumbinary planets are very likely to exist.
\end{abstract}

Keywords. binaries: general, planetary systems: formation

Planet formation around Sun-like stars appears to be a frequent phenomenon. The rapidly increasing number of known planets now enables detailed studies of their statistical properties, which should provide critical elements to disentangle the competing models of "core accretion" (Lissauer \& Stevenson 2007) and "disk instability" (Durisen et al. 2007). In parallel, stellar multiplicity is an ubiquitous outcome of star formation (Duchêne et al. 2007), raising the question of the coexistence of these two phenomena. I present here a brief overview of several key trends connecting planet formation and stellar multiplicity that have been recently unearthed. An expanded version of this work will appear elsewhere (Duchêne, in prep.).

Studies of protoplanetary disks in pre-main sequence multiple systems have revealed that the most massive component of a system is usually associated with the most massive and longestlived disk (see Monin et al. 2007). Considering disks located in multiple systems, the properties of their innermost, planet-forming, regions are indistinguishable from those of single stars (e.g., White \& Ghez 2001, Pascucci et al. 2008). This suggests that the required initial conditions for planet formation are met equally well in the higher-mass component of a multiple system as in single stars, but that lower-mass components may have more adverse initial conditions to form planets. Beyond these overall properties, there is a clear trend for disks in tighter binaries $(a \lesssim 100 \mathrm{AU})$ to have a much shorter lifetime (Cieza et al. 2009). In addition, it is a long-established fact that tighter binaries $(a \lesssim 300 \mathrm{AU}$ ) have much lower (sub)millimeter fluxes than wider binaries and single stars alike (e.g., Andrews \& Williams 2005). Both observations have been interpreted as the natural consequence of severe disk truncation in tight binaries, leading to a general belief that tight binaries are hard-pressed to form planetary systems at all. The situation is not necessarily as dark as it seems, however, as optical depth effects in dense, compact disks could explain their reduced thermal emission. It is also important to note that the few protoplanetary disks encompassing spectroscopic binaries currently known tend to have large (sub)millimeter fluxes (Jensen et al. 1996), similar to those around single stars, and could therefore offer fertile grounds for planets.

Moving on to the next evolutionary stage, let us focus on debris disks as proxies for newly formed planetary systems. Overall, the debris disk phenomenon occurs at a very similar rate in binaries and single stars (see Trilling et al. 2007). A proposed deficit of debris disks among intermediate separation (3-50 AU) binaries has not been confirmed (Plavchan et al. 2009), suggesting that planetesimal disks form in binaries of all separations. In particular, the shortest period bi- 
naries $(a \lesssim 3 \mathrm{AU}$ ) frequently possess debris disks. In summary, the debris disks phenomenon appears to be indifferent to the presence and/or location of stellar companions.

Finally, let us review the connection between mature planetary systems and multiple stellar systems. Binary systems have long been excluded from planet searching surveys for practical reasons but more and more known examples are discovered in follow-up surveys of known exoplanet hosts. Their multiplicity rate is only slightly lower than that of field stars despite selection biases (Mugrauer \& Neuhaüser 2009). Although the stellar companions tend to be at separations larger than $100 \mathrm{AU}$ (Raghavan et al. 2006), there is a growing number of systems with projected separations as close as $20 \mathrm{AU}$, proving that even a relatively tight companion does not preclude planet formation. An early statistical analysis suggested a peculiar mass-period trend for planets in binary systems with respect to planets around single stars (Zucker \& Mazeh 2002), but larger samples have now shown that this is not statistically significant. On the other hand, this larger sample has revealed that planets in tight binaries $(a \lesssim 100 \mathrm{AU})$ all have $M_{p}>M_{J u p}$, whereas planets in wider binaries and single stars are roughly equally split above and below this threshold. In other words, tight binaries only form high-mass planets.

While there are caveats associated to the various studies listed here, not least the fact that each of them considers a different range of stellar masses, it is tempting to try and put together the various pieces of this puzzle. Overall, it seems clear that primary stars in "wide" binaries, say $a \gtrsim 100 \mathrm{AU}$, evolve in the same manner as single stars, presumably through the "core accretion" scenario since disks survive for quite a long time (5-10 Myr). "Intermediate" binaries ( $5 \lesssim a \lesssim 100 \mathrm{AU}$ or so) have protoplanetary disks that are much denser and more compact, have a much shorter survival timescale, and yet form higher-mass planets on average. I propose that these various trends can be accounted for if planet formation in such binaries occurs via a violent process, such as the "disk instability" mechanism. Finally, while no such planet is known to date, the chances that planets exist around "tight" binaries $(a \lesssim 5 \mathrm{AU})$ are quite high. While detecting such systems is a major observational challenge, it is certainly worth attempting to search for them with both direct and indirect techniques.

\section{References}

Andrews, S. M. \& Williams, J. P. 2005, ApJ, 631, 1134

Cieza, L. A. et al. 2009, ApJ (Letters), 696, L84

Duchêne, G. et al. 2007, Protostars and Planets V, 379

Durisen, R. H. et al. 2007, in Protostars and Planets V, 607

Jensen, E. L. N., Mathieu, R. D., \& Fuller, G. A. 1996, ApJ, 458, 312

Lissauer, J. J. \& Stevenson, D. J. 2007, in Protostars and Planets V, 591

Monin, J.-L. et al. 2007, in Protostars and Planets V, 395

Mugrauer, M. \& Neuhäuser, R. 2009, A\&\&A, 494, 373

Pascucci, I. et al. 2008, ApJ, 673, 477

Plavchan, P. et al. 2009, ApJ, 698, 1068

Raghavan, D. et al. 2006, ApJ, 646, 523

Trilling, D. E. et al. 2007, ApJ, 658, 1289

White, R. J. \& Ghez, A. M. 2001, ApJ, 556, 265

Zucker, S. \& Mazeh, T. 2002, ApJ (Letters), 568, L113 